\title{
REMARKS ON THE RELAXATION APPROXIMATION OF THE BURNETT EQUATIONS
}

\author{
SHI JIN* AND MARSHALL SLEMROD ${ }^{\dagger}$
}

0. Introduction. The purpose of this note is to present a short discussion of the relaxation regularization of the Burnett equations, a second order approximation via the Chapman-Enskog expansion on the Boltzmann equation, proposed in [1].

1. A model problem. The classical Chapman-Enskog expansion in term of the Knudsen number on the Boltzmann equation yields the compressible Euler, NavierStokes and Burnett equations in the zeroth, first and second order truncation [15]. However, truncation at the Burnett order introduces instability of the rest state $[3,4]$, violating the second law of thermodynamics. In [1] we introduced a viscoelastic relaxation to regularize the Burnett equations. As an illustrative example for this relaxation procedure, consider the following model problem

$$
\begin{aligned}
& u_{t}+u u_{x}=\epsilon q_{x}, \\
& q_{t}=-\frac{\left(q-u_{x}\right)}{\epsilon},
\end{aligned}
$$

where $\epsilon>0$.

System (1.1)-(1.2) of course can be rewritten as

$$
\begin{gathered}
u_{t}+u u_{x}-\epsilon q_{x}=0 \\
\epsilon q_{t}-u_{x}=-q
\end{gathered}
$$

and is hyperbolic with damping, and the two characteristics are $\left(u \pm \sqrt{u^{2}+4}\right) / 2$. It is easy to argue that the rest state $u=0, q=0$ is linearly stable.

Now consider the Chapman-Enskog expansion for (1.1), (1.2):

$$
q-u_{x}=-\epsilon q_{t}
$$

$$
q-u_{x}=-\epsilon u_{x t}+\text { higher order terms in } \epsilon .
$$

If we substitute (1.6) into the balance law (1.3) we find "Navier-Stokes" order $\epsilon$

$$
u_{t}+u u_{x}=\epsilon u_{x x}
$$

and $u=0$ is stable. However, if we expand to "Burnett" order $\epsilon^{2}$ we see

$$
u_{t}+u u_{x}=\epsilon u_{x x}-\epsilon^{2} u_{x x t} .
$$

*School of Mathematics, Georgia Institute of Technology, Atlanta, GA 30332, U.S.A. Research supported by NSF grant No. 9704957.

†Center for the Mathematical Sciences, University of Wisconsin-Madison, 1308 W. Dayton St., Madison, WI 53715-1149. Vilas Associate, University of Wisconsin-Madison. This research was supported in part by the National Science Foundation grant Nos. DMS-9803223 and DMS-9531845, Office of Naval Research Contract No. N00014-93-1-0015, and a U.S.-Israel Binational Science Foundation Grant. 
A simple linear stability on (1.8) indicates that the "Burnett" term $u_{x x t}$ yields high frequency instability. Since the original system (1.1), (1.2) definitely has $u=$ 0 stable there is an apparent paradox. This phenomenon has been noted for the case of incompressible viscoelastic fluids by Coleman, Duffin, and Mizel [2] and for compressible gas dynamics by Bobylev [3], [4], see also the papers of Rosenau [5], [6].

Of course, the mistake is that (1.8) is not exact and possible large quantities are omitted in the truncation provided by the "Burnett" equation (1.8). At this point there are (at least) two approaches that may be taken. The first was suggested by Rosenau [5]: simply sum the Chapman-Enskog expansion to recover the exact "constitutive relation" for $q$ as a function $u_{x}$. In fact, for a linearized system of equations derived from Grad's 13 moment system of gas dynamics this has been done by Gorban and Karlin [7]. As yet no exact summation has succeeded in non-linear cases. A second approach is perhaps naive but a little thought points to its scientific merit. Specifically if truncation of the Chapman-Enskog expansion beyond NavierStokes order leads to unphysical stability results perhaps system (1.1)-(1.2) which has stable equilibria and is well posed is indeed a better model. But in practice this is not so easily done. Recall that in rarefied gas dynamics we are not given initially a system of partial differential equation but only the Boltzmann equation or some near relative of it. So that in reality (1.2) represents the Boltzmann equation and (1.1) represents the conservation laws of momentum and energy. So reversion back to the fundamental Boltzmann equation is not desirable in the actual problem of gas dynamics. Instead we suggest forming a new system of partial differential equations similar to (1.1)-(1.2), i.e. a relaxation system which when expanded on the Knudsen number $\epsilon$ (i) captures the Chapman-Enskog expansion to the Burnett order, (ii) is hyperbolic (at least locally), (iii) possesses a globally defined "entropy" inequality.

The idea of relaxation approximation for numerical simulation in gas dynamics is of course not new. In [8] a generic method for relaxation of system hyperbolic conservation laws was introduced; a physically natural pressure relation was presented [9]; Grad [10] and later Levermore [11], [12] developed a theory of moment equations. In a similar spirit rational extended thermodynamics [13] by Müller and Ruggeri develops a moment like system of equations except the closure rules are constituted as in continuum mechanics. The main point of our method is that unlike the above mentioned papers it provided item (i) above while not neglecting (ii), (iii), i.e. its Chapman-Enskog expansion exactly matches the classical Chapman-Enskog expansion to Burnett order $\epsilon^{2}$ and hence provides a natural regularization of the Burnett equations. 


\section{The relaxation approximation.}

Denote by

$\begin{array}{ll}I_{1}, I_{2}, I_{3} & \text { 2th coefficient of the Chapman-Enskog expansion for } \mathbf{q} \\ \rho & \text { density } \\ \mathbf{u} & \text { velocity } \\ \mathbf{P} & \text { pressure deviator } \\ p & \text { mean normal pressure } \\ \mathbf{b} & \text { body force } \\ \mathbf{L} & (\text { grad } \mathbf{u})^{T} \\ e & \text { internal energy density } \\ \mathbf{q} & \text { energy flux vector } \\ \theta & \text { temperature } \\ \theta_{i} & i^{t h} \text { coefficient of the Chapman-Enskog expansion for } \mathbf{q} \\ \omega_{i} & i^{\text {th }} \text { coefficient of the Chapman-Enskog expansion for } \mathbf{P} \\ M & \text { Maxwell number } \\ \mu & \text { viscosity } \\ \eta & \text { specific entropy }\end{array}$

Our relaxation approximation is given by

$$
\begin{gathered}
\dot{\rho}+\rho \operatorname{div} \mathbf{u}=0 \\
\rho \dot{\mathbf{u}}+\operatorname{grad} p+\operatorname{div} \mathbf{P}=\rho \mathbf{b}, \\
\mathbf{P}=\mathbf{P}^{T} \\
\dot{\mathbf{P}}-\mathbf{L}^{T} \mathbf{P}-\mathbf{P} \mathbf{L}+\frac{2}{3} \operatorname{tr}(\mathbf{P} \mathbf{L}) \mathbf{I}=-\frac{2 p}{\omega_{2} \mu}\left(\mathbf{P}-\mathbf{P}^{e q}\right), \\
\rho \dot{\mathrm{e}}+p \operatorname{div} \mathbf{u}+\mathbf{P} \cdot \mathbf{S}+\operatorname{div} \mathbf{q}=0, \\
\dot{\mathbf{q}}-\mathbf{L} \mathbf{q}=-\frac{3 M p}{2 \theta_{2} \mu}\left(\mathbf{q}-\mathbf{q}^{e q}\right),
\end{gathered}
$$

where

$$
\mathbf{P}^{e q}=-2 \mu \mathbf{S}+\mathbf{P}_{2}+\mathbf{P}_{3}
$$

$$
\begin{aligned}
\mathbf{P}_{2}= & -\mu \frac{\omega_{1}}{2 p}(\operatorname{div} \mathbf{u}) \mathbf{P}+\frac{\omega_{2} \mu^{\prime}(\theta) \dot{\theta}}{2 p} \mathbf{P} \\
& +\mu^{2} \frac{\omega_{3}}{\rho \theta}\left\{-\operatorname{grad}\left(\frac{\mathbf{q}}{\frac{3}{2} \mu M R}\right)+\frac{1}{3} \operatorname{div}\left(\frac{\mathbf{q}}{\frac{3}{2} \mu M R}\right) \mathbf{I}\right\} \\
& +\mu \frac{\omega_{4}}{\rho p \theta}\left\{-\frac{1}{2} \operatorname{grad} p \otimes\left(\frac{\mathbf{q}}{\frac{3}{2} M R}\right)-\frac{1}{2}\left(\frac{\mathbf{q}}{\frac{3}{2} M R}\right) \otimes \operatorname{grad} p\right.
\end{aligned}
$$




$$
\begin{gathered}
\mathbf{P}_{3}=\mu^{2}\left[\frac{\hat{\omega}_{2}}{p^{2}} \operatorname{tr} \mathbf{S}^{2}+\hat{\omega}_{3} \frac{|\operatorname{grad} \theta|^{2}}{R \rho^{2} \theta^{3}}\right] \mathbf{P}+\mu \frac{\hat{\gamma_{1}}}{p \theta}\left(\dot{\theta}+\frac{2}{3} \theta \operatorname{divu}\right) \mathbf{P} \\
\mathbf{q}^{e q}=-\frac{3}{2} \mu M R \operatorname{grad} \theta+\mathbf{q}_{2}+\mathbf{q}_{3}, \\
\mathbf{q}_{2}=-2 \mu \frac{\theta_{1}}{3 M R \rho \theta}(\operatorname{div} \mathbf{u}) \mathbf{q}+\frac{2 \theta_{2} \dot{\theta} \mu^{\prime}(\theta)}{3 M R \rho \theta} \mathbf{q} \\
-\mu \frac{\theta_{3}}{2 p \rho} \mathbf{P} \operatorname{grad} p-\mu^{2} \frac{\theta_{4}}{2 \rho} \operatorname{div}\left(\frac{\mathbf{P}}{\mu}\right)-\mu \frac{\theta_{5}}{2 \rho \theta} \mathbf{P} \operatorname{grad} \theta, \\
\mathbf{q}_{3}=\mu^{2}\left[\frac{\hat{\theta_{2}}}{p^{2}} \operatorname{tr}^{2}+\hat{\theta}_{3} \frac{|\operatorname{grad} \theta|^{2}}{R \rho^{2} \theta^{3}}\right] \mathbf{q}+\mu \frac{\hat{\lambda_{1}}}{\rho \theta^{2}}\left(\dot{\theta}+\frac{2}{3} \theta \operatorname{div} \mathbf{u}\right)\left(\frac{\mathbf{q}}{\frac{3}{2} M R}\right) .
\end{gathered}
$$

Since the energy equation (2.5) implies that

$$
\dot{\theta}+\frac{2}{3} \theta \operatorname{divu}=\frac{2}{3 \rho R}(-\mathbf{P} \cdot \mathbf{S}-\operatorname{divq})
$$

system (2.1)-(2.6) is local (does not contain $\dot{\theta}$ on the right hand side) after using (2.13). Moreover, (2.13) suggests that $\dot{\theta}+\frac{2}{3} \theta \operatorname{div} \mathbf{u}=O(\mu)$, and $\mathbf{P}_{3}$ and $\mathbf{q}_{3}$ are $O\left(\mu^{3}\right)$, thus belong to the super Burnett order.

We assume $\theta_{3}+\omega_{3}+\omega_{4}=0, \omega_{5}=\frac{\mu^{\prime}(\theta) \theta}{\mu(\theta)} \omega_{3}, \theta_{5}=\bar{\theta}_{5}+\frac{\mu^{\prime}(\theta) \theta}{\mu(\theta)} \omega_{5}, \omega_{3}>0, \theta_{2}>0$, $\bar{\theta}_{5}>0, \bar{\theta}_{5}$ a constant.

It is a relatively easy exercise to see that (2.1)-(2.7) when expanded in powers of $\mu$ (which is proportion to the Knudsen number $\epsilon$ ) agrees with the classical ChapmanEnskog expansion for the Boltzmann equation to Burnett order $\mu^{2}$ as given in [14], [15].

Furthermore, in the case of one dimensional motion $\mathbf{u}=\left(u\left(x_{1}, t\right), 0,0\right), \rho=$ $\rho\left(x_{1}, t\right), \theta=q\left(x_{1}, t\right)$ linearized about $u=0, \rho=\bar{\rho}, \theta=\bar{\theta}$ where $\bar{\rho}, \bar{\theta}$ are constants (2.1)-(2.7) is hyperbolic. Finally we note that the following generalized entropy inequality holds.

3. A generalized entropy inequality. Let $\mathbf{P}, \mathbf{q}$ be given by (2.1)-(2.12) with

$$
\begin{aligned}
& \hat{\lambda_{1}}=-\frac{1}{2} \theta \frac{\partial \theta_{2}}{\partial \theta}-\theta_{2} \theta \frac{\mu^{\prime}(\theta)}{\mu(\theta)}+\frac{3}{2} \theta_{2}, \\
& \hat{\gamma_{1}}=-\frac{1}{2} \theta \frac{\partial \omega_{2}}{\partial \theta}-\omega_{2} \theta \frac{\mu^{\prime}(\theta)}{\mu(\theta)}+\omega_{2},
\end{aligned}
$$


in (2.9), (2.12) respectively. Then the following equality holds:

$$
\begin{aligned}
& \rho\left\{-\eta+\frac{1}{2} \operatorname{tr}\left(\frac{\omega_{2} \mathbf{P}^{2}}{4 \rho p \theta}\right)+\frac{1}{3 M R}\left(\frac{2 \theta_{2}|\mathbf{q}|^{2}}{3 M R \rho^{2} \theta^{3}}\right)\right\}^{\bullet}+\operatorname{div}\left\{\frac{\mathbf{q}}{\theta}+\frac{\omega_{3} \mathbf{P q}}{3 M R \rho \theta^{2}}\right\} \\
= & -\frac{1}{2} \frac{\operatorname{tr} \mathbf{P}^{2}}{\mu \theta}-\frac{2}{3 M R} \frac{|\mathbf{q}|^{2}}{\mu \theta^{2}}+\frac{2 \omega_{2}-\omega_{6}}{4 p \theta} \operatorname{tr}\left(\mathbf{S P}^{2}\right) \\
& +\frac{2}{3 M R}\left(\frac{2 \theta_{2}}{3 M R \rho \theta^{3}}\right) \operatorname{tr}(\mathbf{S q} \otimes \mathbf{q})+\frac{1}{3 M R}\left(-\overline{\theta_{5}}-\omega_{3}+\omega_{3}^{\prime}(\theta) \theta\right) \operatorname{tr}(\mathbf{P g r a d} \theta \otimes \mathbf{q}) \\
& +\left\{\hat{\omega_{2}} \frac{\operatorname{tr} \mathbf{S}^{2}}{p^{2}}+\hat{\omega_{3}} \frac{|\operatorname{grad} \theta|^{2}}{R \rho^{2} \theta^{3}}\right\} \frac{\mu}{R \theta} \operatorname{tr} \mathbf{P}^{2} \\
& +\left\{\hat{\theta_{2}} \frac{\operatorname{tr} \mathbf{S}^{2}}{p^{2}}+\hat{\theta_{3}} \frac{|\operatorname{grad} \theta|^{2}}{R \rho^{2} \theta^{3}}\right\} \mu \frac{|\mathbf{q}|^{2}}{\theta^{2}}\left(\frac{2}{3 M R}\right) .
\end{aligned}
$$

It is then a relatively easy matter to show that the right side of (3.2) is less than or equal to $-\frac{1}{\mu} \mathbf{z} \cdot \mathbf{D z}$ where

$$
\begin{aligned}
& =\left[\begin{array}{ccccc}
\frac{1}{2} & 0 & \frac{\left|\omega_{6}-2 \omega_{2}\right|}{8 \sqrt{2}} & 0 & \frac{1}{2 M} \sqrt{\frac{2}{3}}\left|-\bar{\theta}_{5}-\omega_{3}+\theta \omega_{3}^{\prime}(\theta)\right| \\
0 & 1 & 0 & \frac{1}{3 M} & 0 \\
\frac{\left|\omega_{6}-2 \omega_{2}\right|}{8 \sqrt{2}} & 0 & \hat{\omega}_{2} & 0 & 0 \\
0 & \frac{1}{3 M} & 0 & \hat{\theta_{2}} & 0 \\
\frac{1}{2 M} \sqrt{\frac{2}{3}}\left|-\bar{\theta}_{5}-\omega_{3}+\theta \omega_{3}^{\prime}(\theta)\right| & 0 & 0 & 0 & \frac{\hat{\theta}_{3}}{M}
\end{array}\right], \\
& =\left[\left(\frac{\operatorname{tr} \mathbf{P}^{2}}{\theta}\right)^{1 / 2}, \sqrt{\frac{2}{3}} \frac{|\mathbf{q}|}{\sqrt{M R} \theta}, \mu \frac{\left(\operatorname{tr} \mathbf{S}^{2} \operatorname{tr} \mathbf{P}^{2}\right)^{1 / 2}}{p(R \theta)^{1 / 2}}, \mu \sqrt{\frac{2}{3}} \frac{\left(\operatorname{tr} \mathbf{S}^{2}\right)^{1 / 2}|\mathbf{q}|}{p \theta(M R)^{1 / 2}}, \sqrt{\frac{2}{3}} \frac{|\operatorname{grad}| \theta|\mathbf{q}|}{p \theta^{3 / 2}}\right] .
\end{aligned}
$$

D is positive definite if $\hat{\omega}_{2}>0, \hat{\theta}_{2}>0, \hat{\theta}_{3}>0$ are sufficiently large, $\hat{\omega}_{3}=0$, and $\left|-\bar{\theta}_{5}-\omega_{3}+\theta \omega_{3}^{\prime}(\theta)\right|,\left|\omega_{6}-2 \omega_{3}\right|$ are bounded.

Hence we have produced a global generalized Clausius-Duhem "entropy inequality" for the relaxation system (2.1)-(2.7).

\section{REFERENCES}

[1] Jin, S. AND Slemrod, M., Regularization of the Burnett equations via relaxation, submitted Arch. Rational Mech. Anal.

[2] Coleman, B. D., Duffin, R. J., AND Mizel, V., Instability, uniqueness, and non-existence theorems for the equation $u_{t}=u_{x x}-u_{x t x}$ on a strip, Arch. Rational Mech. Anal., 19 (1965), pp. 100-116.

[3] Bobylev, A. V., On the structure of spatially homogeneous normal solutions of a nonlinear Boltzmann equation for a mixture of gases, Soviet Physics Doklady, 25 (1980), pp. 30-32.

[4] BoBylev, A. V., The Chapman-Enskog and Grad methods for solving the Boltzmann equation, Soviet Physics Doklady, 27 (1), 1982.

[5] Rosenau, P., Extending hydrodynamics via the regularization of the Chapman-Enskog expansion, Phys. Review A, 40 (1989), pp. 7193-7196.

[6] Rosenau, P., Free-energy functionals at the high gradient limit, Phys. Review A, 41 (1990), pp. $2227-2230$. 
[7] Gorban, A. N. And Karlin, I. V., Short wave limit of hydrodynamics: a soluble example, Phys. Review Letters, 77 (1996), pp. 282-285.

[8] JIN, S. AND XIN, Z.P., The relaxation schemes for systems of conservation laws in arbitrary space dimensions, Comm. Pure Appl. Math., 48 (1995), pp. 235-276.

[9] Coquel, F. And Perthame, B., Relaxation of energy and approximate Riemann solvers for general pressure laws in fluid dynamics, SIAM J. Num. Anal., 35 (1998), pp. 2223-2249.

[10] Grad, H., Asymptotic theory of the Boltzmann equation, Physics of Fluids, 6 (1963), pp. 147-181.

[11] Levermore, C.D., Moment closure hierarchies for kinetic theories, J. Stat. Phys., 83 (1996), pp. 1021-1065.

[12] Levermore, C.D. AND MorokofF, W.J., The Gaussian moment closure for gas dynamics, SIAM J. Appl. Math., 59 (1998), pp. 72-96.

[13] Müller, I. AND Ruggeri, T., Rational Extended Thermodynamics, 2nd ed., Springer, 1998.

[14] Ferziger, J. H. AND KAPER, H. G., Mathematical theory of transport processes in gases, Amsterdam: North Holland (1972).

[15] Truesdell, C. And Muncaster, R. G., Fundamentals of Maxwell's Kinetic Theory of a Simple Monatomic Gas, New York: Academic Press (1980). 